\title{
Upregulation of TCF4 expression as a transcriptional target of $\beta$-catenin/p300 complexes during trans-differentiation of endometrial carcinoma cells
}

\author{
Makoto Saegusa $^{1}$, Miki Hashimura ${ }^{1}$, Takeshi Kuwata ${ }^{2}$, Mieko Hamano $^{3}$ and Isao Okayasu ${ }^{1}$ \\ ${ }^{1}$ Department of Pathology, Kitasato University School of Medicine, Kitasato, Sagamihara, Kanagawa, Japan; \\ ${ }^{2}$ Department of Carcinogenesis, The Cancer Institute, Japanese Foundation for Cancer Research, 1-37-1 \\ Kamiikebukuro, Toshima-ku, Tokyo, Japan and ${ }^{3}$ Division of Cell and Tissue Culture, Kitasato University \\ School of Medicine, Kitasato, Sagamihara, Kanagawa, Japan
}

\begin{abstract}
Nuclear stabilization of $\beta$-catenin and its interaction with TCF/LEF factors are key events in transduction of the $\mathrm{Wnt} / \beta$-catenin signal pathway. Our previous study indicated that nuclear $\beta$-catenin accumulation provides an initial signal for trans-differentiation toward the squamoid phenotype of endometrial carcinoma (Em $\mathrm{Ca}$ ) cells in a TCF4-dependent manner, which makes this a possible factor for a positive prognosis. However, little is known about regulation of TCF4 expression in Em Cas. We show here that $\beta$-catenin can directly induce transcription from the TCF4 promoter, the effect being enhanced by the $\mathrm{p} 300$ coactivator. In clinical cases, nuclear $\beta$-catenin accumulation was found to frequently overlap with TCF4 immunoreactivity in morules and surrounding glandular carcinoma lesions, showing a significant positive correlation $(r=0.82, P<0.0001)$, in contrast to areas of squamous metaplasia (SqM) within Em Cas. In cases with coexistence of two squamoid features in transdifferentiated areas, loss of nuclear $\beta$-catenin and TCF4 immunoreactivity was closely related to change in the morphology from the morular to the SqM phenotype. The TCF4 promoter contains a single consensus TCFbinding site that is critical for activation by $\beta$-catenin. The p300 coactivator, in particular $\mathrm{N}$-terminal residues 1 to 670 , appears sufficient to enhance $\beta$-catenin-dependent transcription, again with TCF4-dependence. These findings indicate that a positive feedback loop of TCF4 expression mediated by $\beta$-catenin/p300 may be important for initial steps during trans-differentiation of Em Ca cells. In addition, its downregulation is associated with induction of a more-differentiated squamoid phenotype.

Laboratory Investigation (2005) 85, 768-779, advance online publication, 4 April 2005; doi:10.1038/labinvest.3700273
\end{abstract}

Keywords: $\beta$-catenin; TCF4; $\mathrm{p} 300$; endometrial carcinoma; trans-differentiation

Wnt ligand-Frizzled receptor interactions at the plasma membrane trigger a cytoplasmic signal transduction cascade that propagates to the nucleus via stabilization and release of cytoplasmic $\beta$-catenin. This is closely linked with modulation of numerous processes in development, including differentiation, proliferation, and morphogenesis. ${ }^{1}$ In quiescent cells, the cytoplasmic $\beta$-catenin fraction is at very low levels, because of rapid turnover through the ubiquitin-proteasome pathway promoted by complexes containing the adenomatous polyposis coli (APC)

Correspondence: Dr M Saegusa, MD, Department of Pathology, Kitasato University School of Medicine, 1-15-1 Kitasato, Sagamihara, Kanagawa 228-8555, Japan.

E-mail: msaegusa@med.kitasato-u.ac.jp

Received 16 December 2004; revised 11 February 2005; accepted 14 February 2005; published online 4 April 2005 protein-axin/conductin-glycogen synthase kinase (GSK) $3 \beta$. High levels of $\beta$-catenin due to escape from degradation cause an increase in interaction with the T-cell factor/lymphoid-enhancer factor (TCF/LEF), resulting in inappropriate activation of target genes that mediate the downstream effects of Wnt. ${ }^{2-4}$

In mammals, the TCF/LEF family includes four genes, TCF1, LEF1, TCF3 and TCF4, which display some structural features in common. ${ }^{5,6}$ In each case, the extreme $\mathrm{N}$ terminus harbors a $\beta$-catenin-binding domain, while nearly identical HMG-box domains, recognizing the consensus sequence CTTTG A/T A/T, are located near the $\mathrm{C}$ terminus or in the middle of the TCFs. In the absence of Wnt signals, TCFs interacts with the TLE/Groucho transcriptional corepressor, resulting in repression of TCF-dependent transcription of several genes. ${ }^{7}$ Thus, the role of the family in gene transcription is considered to be as chromosomal 
docking sites for various interaction partners. ${ }^{8}$ In addition, TCF4 and LEF1 have been reported to have different transactivation capacities and distinct spectra of target genes. ${ }^{8}$ For example, LEF1 is not expressed in cells in the normal colon, but is aberrantly activated during colon carcinogenesis, in contrast to TCF4, which plays a central role in normal colonic tissue. ${ }^{9}$

Focal squamous differentiation $(\mathrm{SqD})$ in endometrial carcinomas (Em Cas) may be associated with an increased probability of survival. ${ }^{10,11}$ We have previously demonstrated that mutations in exon 3 of the $\beta$-catenin gene are more frequent $(66.7 \%)$ in $\mathrm{Em}$ Cas with SqD areas than in tumors without such lesions $(12 \%)$, the single nucleotide substitutions being identical between the $\mathrm{SqD}$ areas and the surrounding glandular carcinoma components. ${ }^{12,13}$ Our recent study also indicated that nuclear $\beta$ catenin accumulation is an initial signal for transdifferentiation, in a TCF4-dependent manner. ${ }^{13}$ The available findings allow us to speculate that $\beta$ catenin/TCF4-mediated trans-differentiation may play an important role in Em Ca behavior. However, little is known about the regulatory mechanism of TCF4 during the process. To clarify this question, we here investigated changes in TCF4 expression with reference to overexpression of $\beta$-catenin, using clinical samples and an Em Ca cell line. In addition, the effect of p300, which is utilized as a transcriptional coactivator of $\beta$-catenin, ${ }^{14}$ was also examined.

\section{Materials and methods}

\section{Clinical Cases}

Histological findings were reviewed for 223 hysterectomy specimens of endometrioid type Em Cas in the case records of Kitasato University Hospital for the period from 1992 to 2004, according to the criteria of the World Health Organization histological classification (1994). Areas of squamous differentiation (SqD) within tumors were subdivided into two categories, morules and foci of squamous metaplasia (SqM), as described previously. ${ }^{13}$ Briefly, the former were defined as areas consisting of spindle-shaped cells forming growth sheets (Figure 1A) and the latter featured cells with abundant eosinophilic cytoplasm and clear intercellular bridging (Figure 7a). The investigated tumors with SqD areas comprised 59 grade (G) 1 or 2, but no G3 carcinomas, including 33 cases with morules and 26 with SqM foci. Of these, 20 demonstrated coexistence of both $\mathrm{SqD}$ phenotypes, but the majority had either morule or SqM phenotypes. Such cases were therefore categorized on the basis of the predominant type (lesions with at least $70 \%$ of one or the other), 14 into the morule group and six as having SqM areas. A total of 30 cases, including 11 with morules and 19 with SqM foci, overlapped with those used in our previous study. ${ }^{13}$ In addition, 22 samples of non-neoplastic endometrial lesions adjacent to carcinomas were investigated. All tissues were routinely fixed in $10 \%$ formalin and embedded in paraffin wax.

\section{Antibodies}

Used in this study were anti- $\beta$-catenin mouse monoclonal (clone 14, Transduction Lab., Lexington, KY, USA), anti- $\beta$-catenin rabbit polyclonal (Upstate Biotech., Lake Placid, NY, USA), anti-TCF4 mouse monoclonal (clone 6H5-3, Upstate Biotech.), antip300 (C-20) rabbit polyclonal (sc-585, Santa Cruz
HE
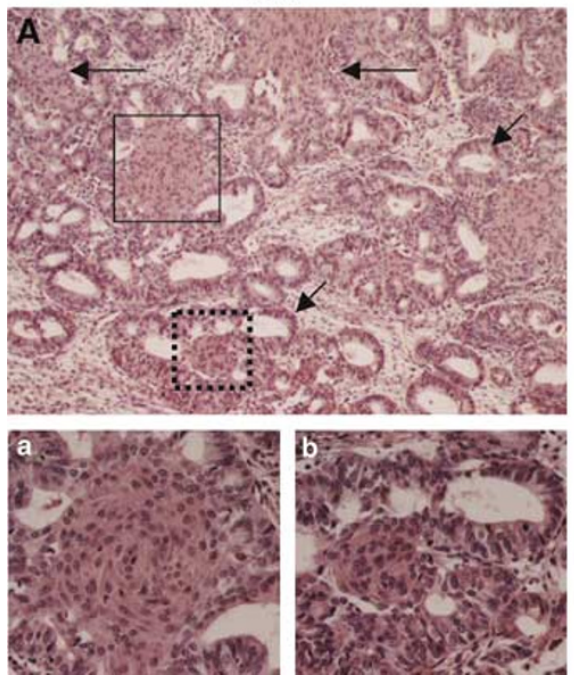
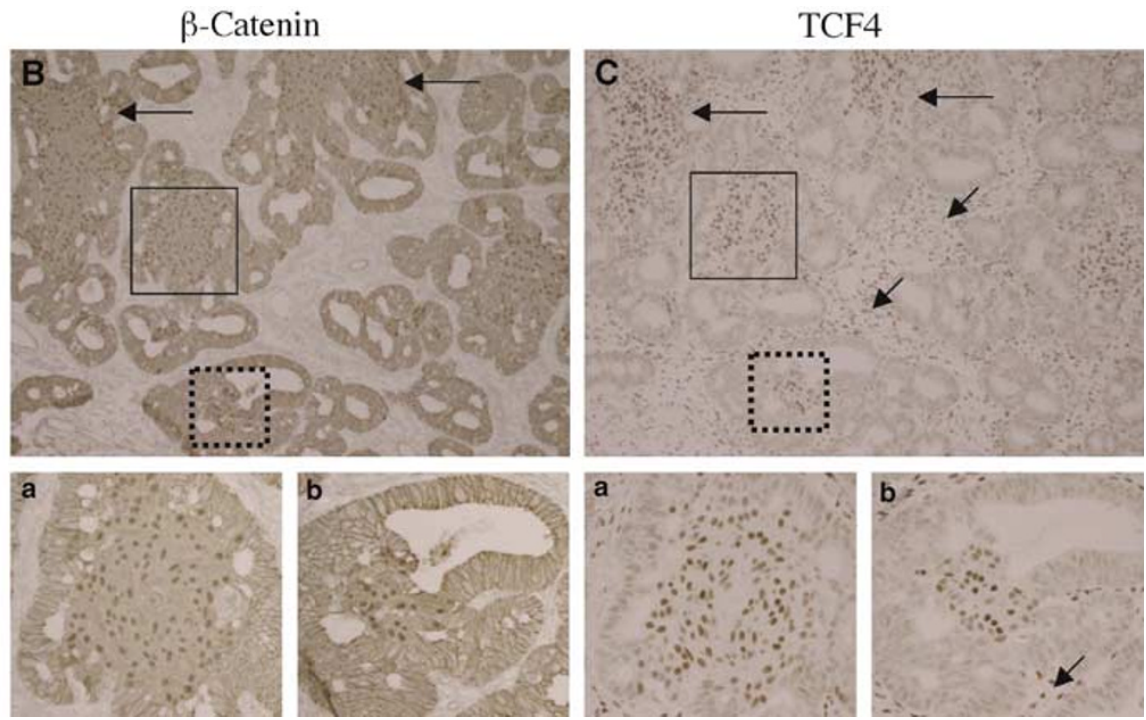

Figure 1 Serial sections of Em Cas with morules. Boxes with solid and dotted outlines enclose a morule and a Ca-around SqD (also indicated by short arrows in (A) lesion magnified in (a) and (b), respectively. Staining is for H\&E (A), and immunohistochemically for $\beta$ catenin (B), and TCF4 (C). Note the frequent nuclear staining for $\beta$-catenin and TCF4 in the morule (indicated by long arrows), as well as the small morular nest in the Ca-around SqD. TCF4 also reacts diffusely with stromal components (indicated by short arrows). Original magnification, $\times 100(\mathrm{~A}-\mathrm{C}) ; \times 400(\mathrm{a}, \mathrm{b})$. 
Biotechnology, Santa Cruz, CA, USA), anti-Flag M2 mouse monoclonal (Sigma Chemical Co., St Louis, MO, USA), anti-phospho-GSK3 $\beta$ (Ser9) rabbit polyclonal (Cell Signaling Technol, Beverly, MA, USA), anti-GSK3 $\beta$ mouse monoclonal (Transduction Lab), anti-cyclin D1 mouse monoclonal (sc-8396, Santa Cruz Biotech.), anti-HA rabbit polyclonal (Y-11, Santa Cruz Biotech.), and anti- $\beta$-actin mouse monoclonal antibodies (Sigma Chemical Co.).

\section{Immunohistochemistry and Evaluation for Staining}

Immunohistochemistry for $\beta$-catenin and TCF4 was performed using new sets of serial sections of all Em Ca tissues, by a combination of microwave-oven heating and standard streptavidin-biotin-peroxidase complex (LSAB kit, Dako, Copenhagen, Denmark) methods. Briefly, slides were heated in $10 \mathrm{mM}$ citrate buffer ( $\mathrm{pH}$ 6.0) for two 10-min cycles using a microwave oven and then incubated overnight at $4^{\circ} \mathrm{C}$ with primary antibodies. To confirm the specificity of binding, normal mouse serum was supplied instead of primary antibody as a negative control.

To evaluate immunohistochemical findings, tumor lesions were subdivided into three areas: SqD, and glandular carcinoma lesions around and distant from the $\mathrm{SqD}$ (Ca-around $\mathrm{SqD}$ and Ca-distant $\mathrm{SqD}$, respectively) Briefly, the Ca-around $\mathrm{SqD}$ was defined as glandular carcinoma elements adjacent to $\mathrm{SqD}$ areas. Such lesions occasionally included small morular foci (Figure 1A). In contrast, the Ca-distant SqD was designated as glandular components without any $\mathrm{SqD}$ areas when examination was performed using low-power $(\times 4$ objective and $\times 10$ ocular $)$ magnification. To determine percentage labeling indices (LIs) for nuclear $\beta$-catenin, immunopositive nuclei were counted for at least 1000 tumor cells in five randomly selected fields each for Ca-around SqD, Ca-distant $\mathrm{SqD}$ and non-neoplastic epithelial (Normal) lesions, and at least 700 cells in all of the morules or the SqM areas, respectively, within tumors in each case, using high-power $(\times 40$ objective and $\times 10$ ocular) magnification. Scoring for TCF4 immunoreactivity was also performed according to the method described by Okudela et al. ${ }^{15}$ Briefly, the percentage of immunopositive cells in the total tumor cell population was subdivided into five categories as follows; 0, all negative; 0.5 , very focal staining $(<5 \%$ positive cells); 1.0 , small areas $(<30 \%)$ of staining with modest immunointensity; 1.5, similar areas with strong immunointensity; 2.0, moderate areas $(<60 \%)$ with modest staining; 2.5 , similar areas with strong staining; 3.0 , diffuse $(<100 \%)$ modest staining; 3.5, diffuse strong staining.

\section{Sequencing}

Genomic DNA was extracted from $4-\mu \mathrm{m}$ thick paraffin wax sections of clinical samples, using proteinase K/phenol-chloroform methods. Sections were reviewed microscopically, and areas with at least $80 \%$ tumor tissues were microdissected, and then exon 3 of the $\beta$-catenin gene was amplified and sequenced, as described previously. ${ }^{12,13,16}$

\section{Plasmids}

The expression vectors for an active form of $\beta$-catenin containing a deleted Ser-45 (pcDNA3. 1 - $\beta$-cat $\triangle \mathrm{S} 45$ ) and the TCF4 lacking 30 amino acids of $\beta$-catenin-binding site in its N-terminus (pcDNA3.1-TCF4 $\triangle$ N30) were as described previously. ${ }^{13}$ HA-tagged $\beta$-cat $\triangle S 45$ was generated with pcDNA3.1- $\beta$-cat $\triangle \mathrm{S} 45$ as a template by PCR using HA- $\beta$-cat- $5^{\prime}-$ and pcDNA3.1/BGH reverse primers (Invitrogen, Carlsbad, CA, USA). The expected size of PCR product was purified and cloned into pcDNA3.1 (Invitrogen), and the sequence was verified by DNA sequencing. pM- $\beta$-cat $\triangle \mathrm{S} 45$ was constructed by inserting the $\beta$-cat $\triangle S 45$ fragment into the pM DNA-BD vector (BD Biosciences Clontech, Worcester, MA, USA) for one-hybrid assays. pG5Luc, in which the CAT gene in pG5CAT reporter vector (BD Biosciences Clontech) was replaced by the luciferase gene, was applied for this purpose.

The TCF4 promoter reporter construct (pGL[-1306] TCF4-Luc) was kindly provided by Dr K Engeland (University of Leipzig, Leipzig, Germany). The pGL-[-445] TCF4-Luc and pGL-[-445] TCF4mutant-Luc were generated with pGL-[-1306] TCF4-Luc by PCR using -445TCF4-Luc-5' - and -445TCF4mutLuc-5'-primers, respectively, and the GL primer 2 (Promega, Madison, WI, USA). The PCR products were cloned into pGL3-basic (Promega) and the sequences were verified by DNA sequencing. pGL[-252] TCF4-Luc was constructed by inserting the BamHI/NCoI fragment (252 bp) of pGL-[-1306] TCF4Luc into pGL3-basic.

The pCI-Flag-tagged p300 construct for full-length p300, p300N for N-terminal residues 1-670, p300 M for middle portion residues 671-1194, and p300C for C-terminal residues 1136-2414 (Figure 4a), were from Dr RH Goodman (Oregon Health Sciences University, Portland, OR, USA) and Dr Y Nakatani (Dana-Farber Cancer Institute, Boston, MA, USA).

The sequences of the PCR primers used in this study were as follows: HA- $\beta$-cat-5'-primer, $5^{\prime}$-GCGAA TTCACCATGTACCCATACGATGTTCCAGATTACGC TGCTACTCAAGCTGATTTG-3'; - -445TCF4-Luc-5'primer, GTCAATCAGCGCCGCCTTTGAACTGAAAA GCTCTCAGTCTAAC-3'; and the -445TCF4mut-Luc5'-primer, 5'-GTCAATCAGCGCCGCCTTTCGCCTGA AAAGCTCTCAGTCTAAC-3' .

\section{Cell Lines and Establishment of Cells Stably Expressing $\beta$-cat $\triangle S 45$}

The Ishikawa Em Ca cell line, ${ }^{17}$ homozygous for $\beta$-catenin wild-type alleles, ${ }^{13}$ was maintained in Eagle's MEM with 10\% bovine calf serum. 
To establish an Em cell line stably expressing the active form of $\beta$-catenin, the pcDNA-HA- $\beta$-cat $\triangle \mathrm{S} 45$ construct or empty pcDNA3.1 plasmids were transfected into Ishikawa cells using LipofectAMINE PLUS (Invitrogen). After approximately 2 weeks of culture in the presence of $1 \mathrm{mg} / \mathrm{ml}$ of Geneticin (GIBCO BRL, Grand Island, NY, USA), the individual colonies were cloned and screened for their $\beta$-cat $\triangle \mathrm{S} 45$ expression with an anti-HA polyclonal antibody (Santa Cruz Biotech.). Transcriptional activity of exogenous active $\beta$-cat $\triangle \mathrm{S} 45$ was evaluated by Top- and Fop-FLASH reporter assays (Upstate Biotech.).

\section{Transient Transfection and Luciferase Reporter Assay}

Transfection was carried out using LipofectAMINE PLUS (Invitrogen), in duplicate or triplicate, in accordance with the manufacturer's instructions. All reporter assays were carried out with 24-well plates and $0.4 \mu \mathrm{g}$ of total plasmids. The pRL-TK plasmid (Promega) was used to normalize for transfection efficiency. Luciferase activity was assayed $24 \mathrm{~h}$ after transfection, using the Dual-luciferase reporter assay system (Promega).

\section{RT-PCR}

Total RNA was extracted from cells using Isogen (Nippon Gene Co., Tokyo, Japan) and cDNA was synthesized from $2 \mu \mathrm{g}$ of total RNA using RAV-2 reverse transcriptase (Takara, Shiga, Japan) in the presence of random primers (Takara). For investigation of TCF4 mRNA expression, amplification was carried out in the PCR exponential phase (20-25 cycles) to arrow comparison among cDNAs synthesized from identical reactions. Primer sequences were as follows: TCF4 exon 1, 5' $5^{\prime}$ AATCAAAACAGC TCCTCCG-3' (sense); and TCF4 exon 3, 5'-ATGATG AAGGGGTAGCCG-3' (anti-sense). The GAPDH gene was also amplified as an internal control, as described previously. ${ }^{16}$

\section{Immunofluorescence}

Monolayers were fixed with $3.7 \%$ paraformaldehyde for $10 \mathrm{~min}$ and permeabilized in $0.2 \%$ Triton X-100 for $10 \mathrm{~min}$. At $40 \mathrm{~h}$ after transient cotransfection of $\beta$-catenin and p300 constructs or treatment with either $30 \mathrm{mM} \mathrm{NaCl}$ or $30 \mathrm{mM} \mathrm{LiCl} \mathrm{(Sigma}$ Chemical Co.), cells were stained with primary antibodies. The secondary antibodies were FITClabeled anti-mouse or rabbit IgGs (Molecular Probe, Leiden, Netherlands).

\section{Western Blot Assay and Immunoprecipitation}

Total cellular proteins were isolated using RIPA buffer (50 mM Tris-HCl (pH 7.2), 1\% NP-40, 0.5\% sodium deoxycholate, $0.1 \%$ SDS) containing protease inhibitor (phenylmethylsulfony fluide). Cytoplasmic fractions were also prepared according to the protocol described previously, ${ }^{18}$ with minor modification. Briefly, cells were scraped off the plates and lysed with a hypotonic lysis buffer (15 $\mathrm{mM} \mathrm{NH}_{4} \mathrm{Cl}, 14 \mathrm{mM} \mathrm{NaHCO}_{3}$, and $0.1 \mathrm{mM}$ EDTA) for $30 \mathrm{~min}$ on ice. After passage through a 20-gauge needle (10 times), the lysates were centrifuged $(15000 \mathrm{~g})$ and supernatants were collected as cytoplasmic fractions. Aliquots of 1-20 $\mu$ g proteins were resolved by SDS-PAGE, transferred to membranes, and detected with the ECL detection system (Amersham Pharmacia Biotech, Tokyo, Japan).

For immunoprecipitation assays, $1 \mu \mathrm{g}$ of pcDNAHA- $\beta$-cat $\triangle \mathrm{S} 45$, together with $3 \mu \mathrm{g}$ of pCI-Flagp300N, p300 M, or p300C, were transfected into $2 \times 10^{6}$ Ishikawa cells, seeded into $10-\mathrm{cm}$ dishes. Cells were harvested $40 \mathrm{~h}$ later and lysed with TNE buffer (10 mM Tris-Cl pH 7.6, $150 \mathrm{mM} \mathrm{NaCl,} \mathrm{1 \%} \mathrm{NP-}$ 40, $1 \mathrm{mM}$ EDTA) containing protease inhibitor. Cell lysates were cleared and incubated with anti-Flag
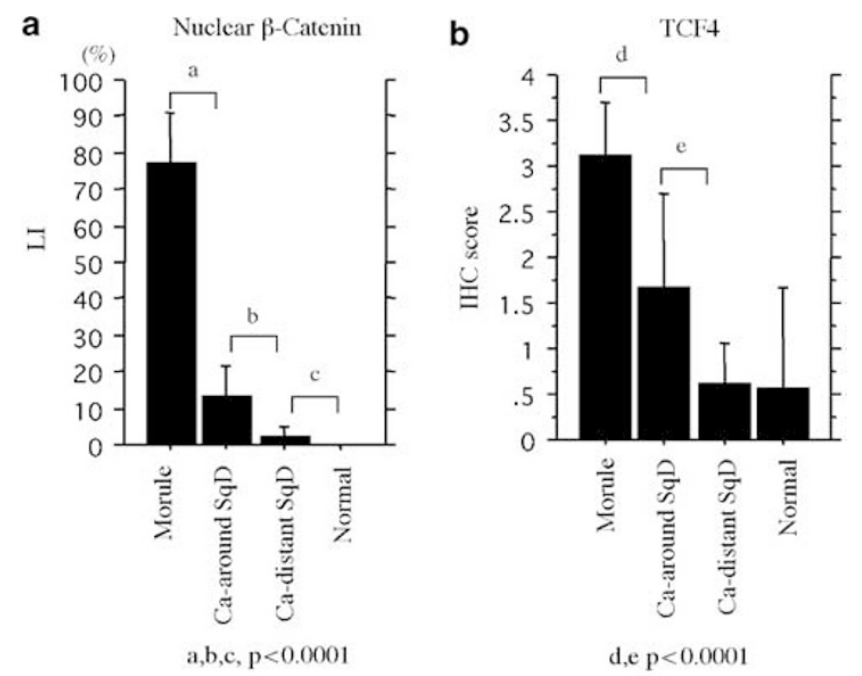

c

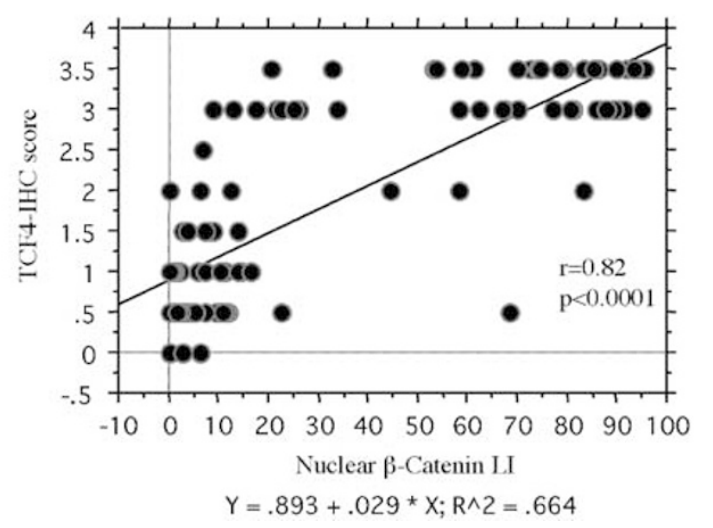

Figure 2 (a, b) LIs and IHC scores for nuclear $\beta$-catenin (a) and TCF4 (b) for morules, Ca-around SqD, Ca-distant SqD and Normal lesions in Em Cas. The data are means \pm s.d. values. (c) Correlations between nuclear $\beta$-catenin LIs and TCF4-IHC scores for Em Cas with morules. r, Pearson's correlation coefficient. 
M2 monoclonal antibodies (Sigma Chemical Co.), followed by incubation with Protein G-Sepharose (Amersham Pharmacia Biotech). Western blotting was subsequently performed with anti-HA polyclonal antibodies (Santa Cruz Biotech.).

\section{Statistics}

Comparative data were analyzed using the MannWhitney $U$-test, and the Pearson's correlation coefficient. The cutoff for statistical significance was set as $P<0.05$.

\section{Results}

\section{Immunohistochemical Findings for Em Cas with Morules}

Mutations in exon 3 of the $\beta$-catenin gene were detected in $23(69.7 \%)$ of 33 informative Em Cas with morules, being closely associated with nuclear accumulation (data not shown), in line with our previous study. ${ }^{12,13}$
Nuclear $\beta$-catenin accumulation was frequent in morules and the Ca-around SqD including small morular nests (Figure 1B), in contrast to the focal staining or absence in Ca-distant SqD and Normal lesions. The immunoreactivity appeared to overlap with nuclear $\beta$-catenin-positive epithelial components (Figure 1C), in addition to strong and diffuse nuclear TCF4 immunoreactivity in stromal components, although the immunoreactions were relatively weak and sporadic in Ca-distant SqD and Normal.

Average labeling indices (LIs) for nuclear $\beta$ catenin and immunohistochemical (IHC) scores for TCF4 showed significant stepwise decrease from morules, through Ca-around $\mathrm{SqD}$ and Ca-distant $\mathrm{SqD}$, to Normal lesions (Figure 2a and b). There was a positive correlation between nuclear $\beta$-catenin LIs and TCF4 IHC scores (Figure 2c).

\section{Upregulation of TCF4 Expression by $\beta$-Catenin}

On treatment of Ishikawa cells with $\mathrm{LiCl}$, an inhibitor of GSK3 $\beta,{ }^{19}$ TCF4 expression was induced a
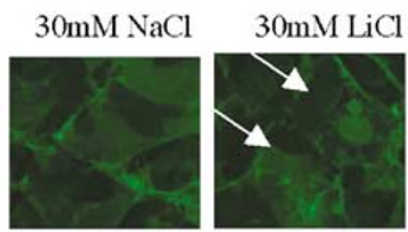

RT-PCR

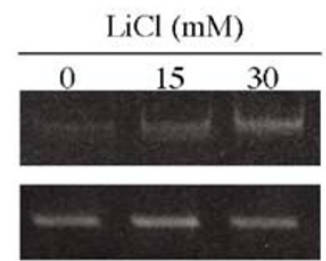

TCF4

GAPDH

Western blot

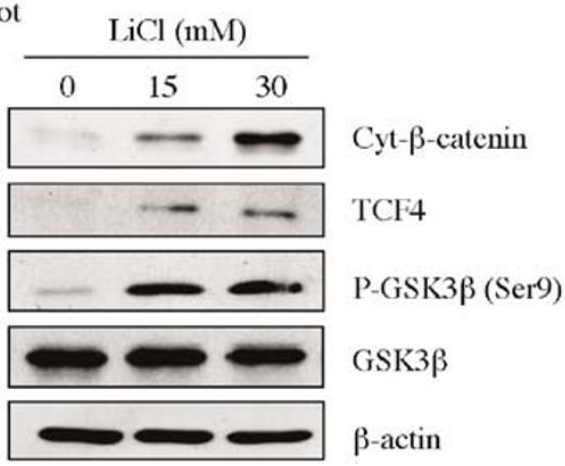

b Ish-HA- $\beta$-cat $\# 25$
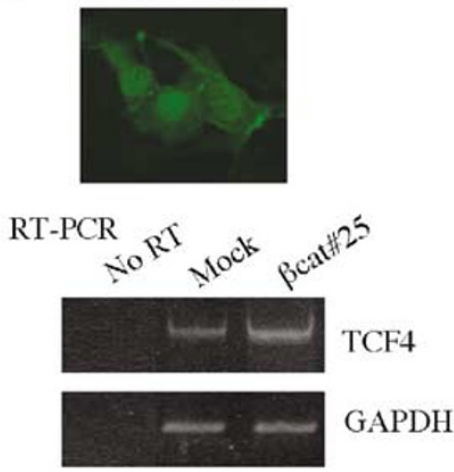

Western blot

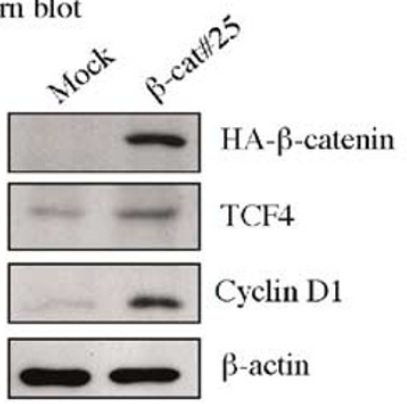

C

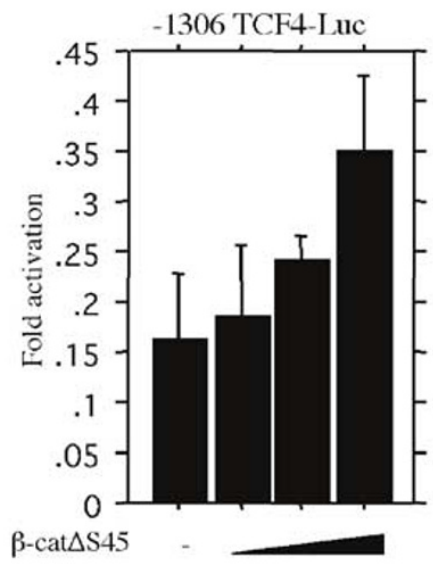

Figure 3 Induction of TCF4 mRNA and protein by stabilization of $\beta$-catenin. (a) After $48 \mathrm{~h}$ of treatment with $30 \mathrm{mM} \mathrm{LiCl}$ or $\mathrm{NaCl}$ as a negative control, Ishikawa cells were stained with anti- $\beta$-catenin mouse monoclonal antibodies (Transduction Lab). Note the nuclear $\beta$ catenin accumulation (indicated by arrows) induced by $\mathrm{LiCl}$ but not $\mathrm{NaCl}$ treatment. Note the RT-PCR findings for TCF4 mRNA and Western blot analyses for cytoplasmic (Cyt) fraction of the $\beta$-catenin, TCF4, the Ser9-phosphorylated form (P) of GSK3 $\beta$, and total GSK3 $\beta$ in Ishikawa cells after 15 and $30 \mathrm{mM} \mathrm{LiCl}$ treatment for $24 \mathrm{~h}$. GAPDH mRNA for RT-PCR and $\beta$-actin for Western blot assays were used as internal controls. (b) Ishikawa cells stably expressing HA- $\beta$-cat $\triangle \mathrm{S} 45$ (Ish-HA- $\beta$-cat\#25). Note the nuclear $\beta$-catenin staining detected with the anti-HA rabbit polyclonal antibody. Both RT-PCR and Western blot assays demonstrate an increase in TCF4 expression in IshHA- $\beta$-cat\#25 as compared to the mock case. Note the increased expression of cyclin D1 proteins in Ish-HA- $\beta$-cat\#25. No RT-reaction samples were also applied as negative control. (c) Ishikawa cells were transfected with $100 \mathrm{ng}$ of -1306 TCF4-Luc and various amounts $(0,50,100,250 \mathrm{ng})$ of $\beta$-cat $\triangle \mathrm{S} 45$, together with the pRL-TK plasmid (50 ng). Relative activities were derived from arbitrary light units of luciferase activity normalized for pRL-TK activity. Data are means \pm s.d. values. 
at both mRNA and protein levels, together with accumulation of nuclear and cytoplasmic $\beta$-catenin and phophorylation at the Ser-9 of GSK3 $\beta$ (Figure 3a). Ishikawa cells stably expressing $H A-\beta$-cat $\triangle \mathrm{S} 45$ constructs showed active $\beta$-catenin-dependent transcription determined with Top- and Fop-FLASH

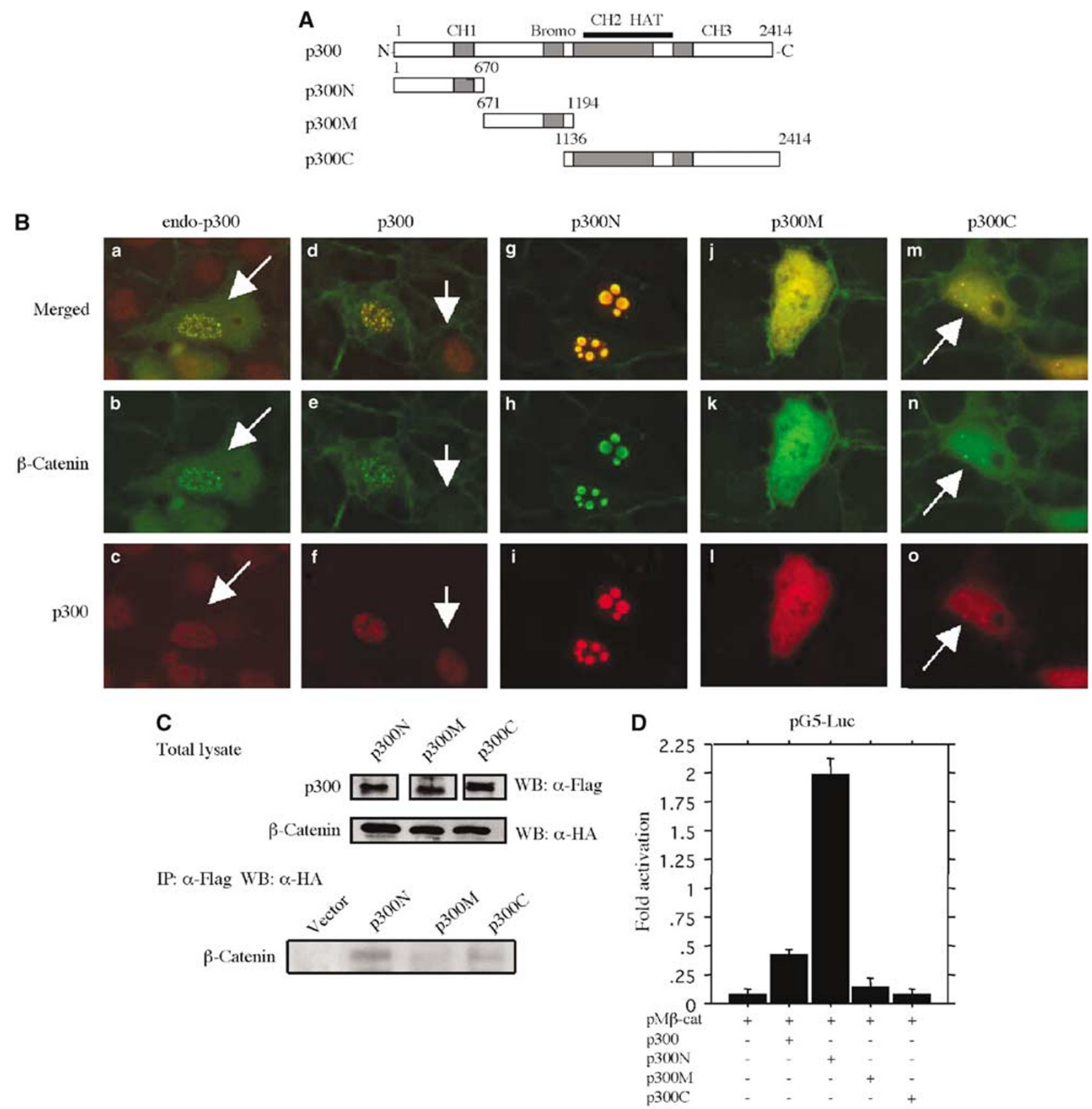

Figure 4 Interaction between $\beta$-catenin and p300. (A) Schematic representation of full-length p300 and the truncated forms (p300N, p300 M, and p300C). The cysteine/histidine-rich elements $\mathrm{CH} 1, \mathrm{CH} 2$, and $\mathrm{CH} 3$, the bromo domain (gray boxes), and the HAT domain (black bar) are denoted. (B) Colocalization of $\beta$-catenin and p300 in Ishikawa cells. (a-c) After transfection of $\beta$-cat $\triangle \mathrm{S} 45$, cells were stained with anti- $\beta$-catenin mouse monoclonal (Transduction Lab) and anti-p300 rabbit polyclonal antibodies. Colocalization of exogenous $\beta$-catenin and endogenous p300 forms is evident in speckle shaped nuclear bodies (indicated by arrows). (d-o) After cotransfection of $\beta$-cat $\triangle \mathrm{S} 45$ and either Flag-p300 (d-f), p300N (g-i), p300 M (j-l), or p300C (m-o), respectively, cells were stained with anti- $\beta$-catenin rabbit polyclonal (Upstate Biotech.) and anti-Flag M2 mouse monoclonal antibodies. Note the enlarged nuclear dots formed by colocalization of $\beta$-cat $\triangle \mathrm{S} 45$ and p300N constructs (g-i), in contrast to the speckle patterns for $\beta$-cat $\triangle \mathrm{S} 45$ and p300 (d-f, cells expressing p300 alone indicated by arrow). Note the diffuse nuclear/cytoplasmic staining with or without a few nuclear aggregates (m-o, indicated by arrow) when combined with either p300 M (j-l) or p300C (m-o). (C) Coimmunoprecipitation of $\beta$-cateinin and p300. Ishikawa cells were transfected with expression plasmid for HA- $\beta$-cat $\triangle \mathrm{S} 45$ and either Flag-p300N, p300 M, or p300C. WB, western blot; IP, immunoprecipitation. (D) Ishikawa cells were transfected with $100 \mathrm{ng}$ of pG5-Luc, $100 \mathrm{ng}$ of pM $\beta$-cat, and $150 \mathrm{ng}$ of either p300, p300N, p300 M, or p300C, respectively, together with the pRL-TK plasmid (50 ng). Relative activities were derived from arbitrary light units of luciferase activity normalized for pRL-TK activity. Data are means \pm s.d. values. 
reporter constructs (data not shown). TCF4 mRNA and protein expression, as well as cyclin D1 protein, which is known as a target gene of $\beta$-catenin, ${ }^{20}$ were also increased as compared to the mock case (Figure $3 b$ ).

The TCF 4 promoter construct containing the 1306 upstream region (-1306 TCF4-Luc) was activated by transfection of $\beta$-cat $\triangle S 45$, in a dose-dependent manner (Figure 3c).

\section{Enhancement of TCF4 Expression by $\beta$-Catenin/p300 Complexes}

Since p300 has been shown to potentiate transcriptional activation of $\beta$-catenin-responsive genes, ${ }^{14}$ we firstly tested whether it influences $\beta$-catenin-dependent transcription in Ishikawa cells. As shown in Figure 4B, colocalization of exogenous $\beta$-catenin and endogenous p300 was found in speckle patterns. A similar picture was also observed when cells were cotransfected with $\beta$-cat $\triangle \mathrm{S} 45$ and Flagp300. Cotransfection with Flag-p300N-terminus constructs resulted in formation of several enlarged dots in nuclei, whereas diffuse nuclear/cytoplasmic staining, with or without formation of nuclear aggregates, was observed after cotransfection with either Flag-p300M or Flag-p300C. An apparent interaction between $\beta$-catenin and p300N was also demonstrated by coimmunoprecipitation from lysates of cells cotransfected with HA- $\beta$-cat $\triangle \mathrm{S} 45$ and Flag-p300N, in contrast to the absent or weak reactions with p300 $\mathrm{M}$ and p300C (Figure 4c). Onehybrid assays revealed the pG5-Luc reporter activity to be raised 3-4-fold by cotransfection of the DNABD-fused full-length $\beta$-catenin fragment (pM $\beta$-cat) and p300, but increased up to 20 -fold by the combination with p300N. Little if any further increase in the reporter activity was conferred by coexpression of either p300 M or p300C (Figure 4d).

As shown in Figure 5, coexpression of p300N and $\beta$-catenin, along with the -1306 TCF4 promoter construct, resulted in an about 12-fold increase in luciferase activity, in a TCF4-dependent manner.

\section{Identification of TCF-Binding Site in the TCF Promoter}

A search of the TCF4 promoter (GenBank Accession Number AF522996) for potential TCF-binding sites revealed the presence of a CTTTGAA sequence at position -430 to -424 . To determine the functional significance of this putative TCF binding sequence, we employed a 445-bp proximal promoter construct. A five-fold induction in the promoter activity was observed with cotransfection of $\beta$-catenin and p300N, but this effect disappeared with the 252-bp construct, in which the putative site was deleted (Figure 6a). By introducing triple mutations in the context of the -455 TCF 4 promoter, transactivation by $\beta$-catenin/p300N was abolished (Figure 6b). a

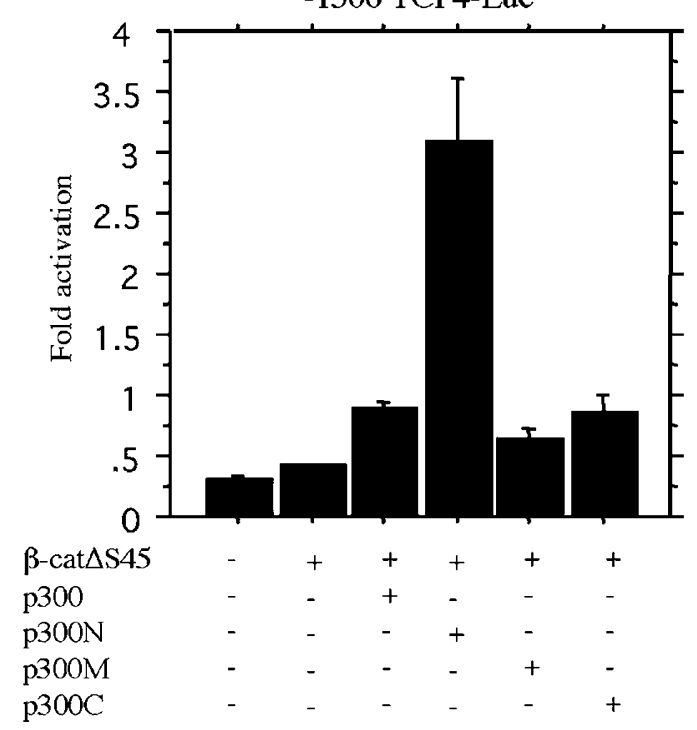

b

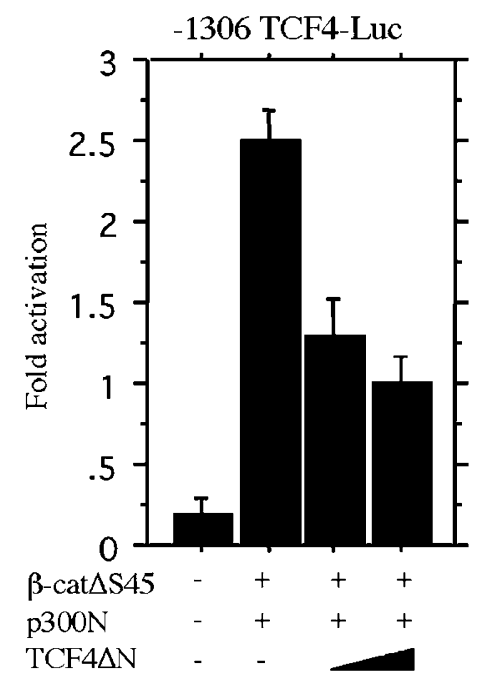

Figure 5 Enhanced transcriptional activity of the TCF4 promoter with the $\beta$-catenin $/ \mathrm{p} 300 \mathrm{~N}$ complex. (a) Ishikawa cells were transfected with $100 \mathrm{ng}$ of -1306 TCF4-Luc, $100 \mathrm{ng}$ of $\beta$-cat $\triangle \mathrm{S} 45$, and $150 \mathrm{ng}$ of either p300 or its truncated forms, together with the pRL-TK plasmid (50 ng). The data are means \pm s.d. values. (b) Ishikawa cells were transfected with $100 \mathrm{ng}$ of -1306 TCF4-Luc, $100 \mathrm{ng}$ of $\beta$-cat $\triangle \mathrm{S} 45,50 \mathrm{ng}$ of $\mathrm{p} 300 \mathrm{~N}$, and various amounts (50, $100 \mathrm{ng}$ ) of TCF $\triangle \triangle \mathrm{N}$. Relative activities were derived from arbitrary light units of luciferase activity normalized for pRL-TK activity. Data are means \pm s.d. values.

\section{Downregulation of $\beta$-Catenin and TCF4 Expression in SqM Areas of Em Cas}

Mutations in exon 3 of $\beta$-catenin gene were also detected in eight (25.8\%) of 25 informative Em Cas with SqM areas, demonstrating sporadic and weak nuclear immunoreactions for $\beta$-catenin, as well as TCF4. The LI values revealed stepwise decreases in values from $\mathrm{SqM}$, through Ca-around $\mathrm{SqD}$ and Cadistant $\mathrm{SqD}$, to Normal lesions, although the two did not show any correlation (Figure 7). 
a
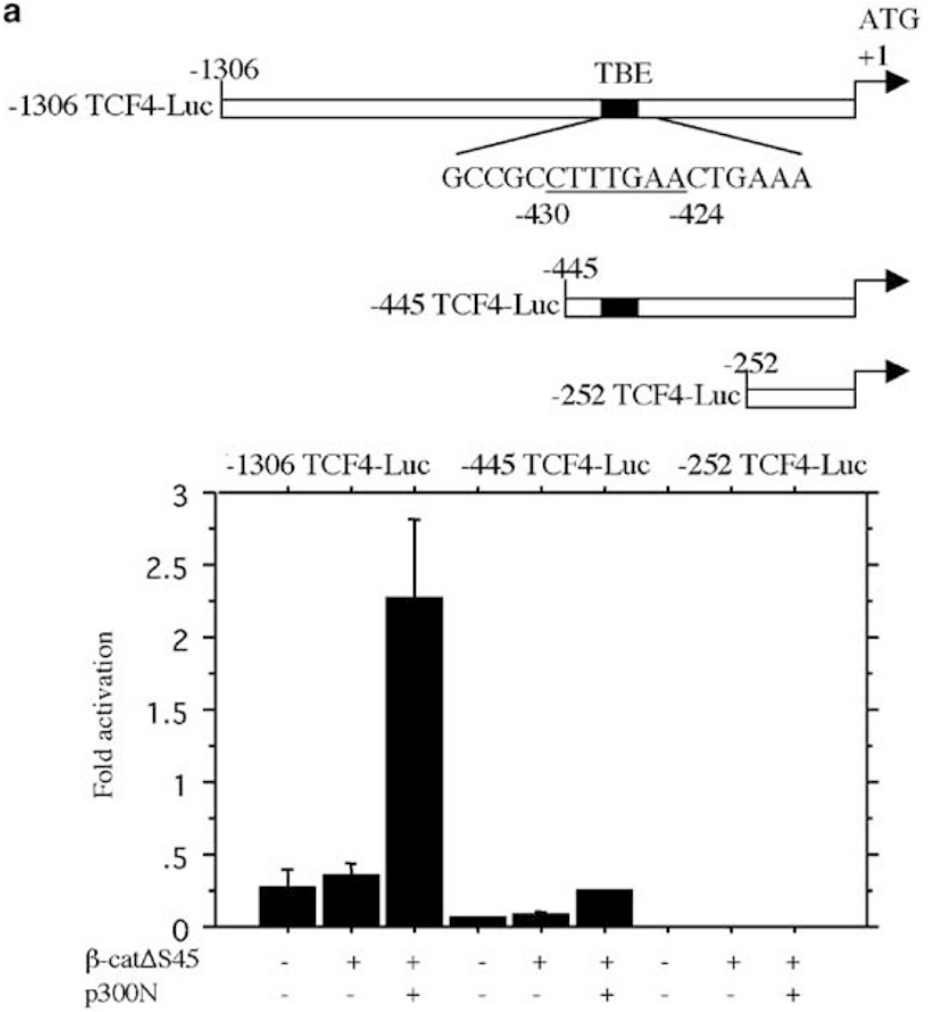

b
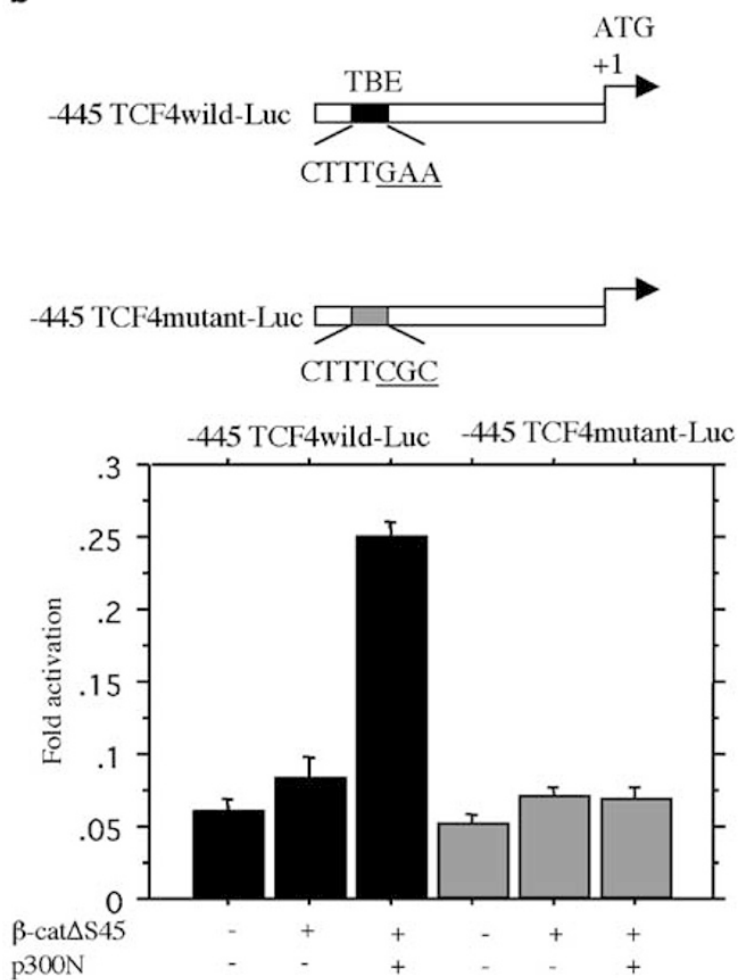

Figure 6 (a) Schematic representation of a putative TCF/LEF binding element located at position -430 bp from the transcriptional start site and deletion constructs ( 445 and $252 \mathrm{bp}$ ) of the TCF4 promoter in the pGL3-basic luciferase reporter plasmid. Ishikawa cells were transfected with $100 \mathrm{ng}$ of $-1306 \mathrm{TCF} 4$-Luc, $-445 \mathrm{TCF} 4$-Luc, or $-252 \mathrm{TCF} 4$-Luc, respectively, and $100 \mathrm{ng}$ of $\beta$-cat $\triangle \mathrm{S} 45$, and/or $150 \mathrm{ng}$ of p300N, together with the pRL-TK plasmid (50 ng). Relative activities were derived from arbitrary light units of luciferase activity normalized for pRL-TK activity. Data are means \pm s.d, values. (b) Triple mutations known to abolish TCF binding were introduced into the TCF recognizing site in the context of the -445 TCF4 promoter. Ishikawa cells were transfected with $100 \mathrm{ng}$ of wild or mutated -445 TCF4-Luc, and $100 \mathrm{ng}$ of $\beta$-cat $\triangle \mathrm{S} 45$, and $150 \mathrm{ng}$ of p300N, together with the pRL-TK plasmid (50 ng). Data are means \pm s.d. values.

In 14 cases with coexistence of two phenotypes in trans-differentiated areas, morular cells showed a sequential change in the morphology to SqM cells, in line with loss of nuclear $\beta$-catenin and TCF4 immunoreactivities. Such cases showed a positive correlation between the two molecules (Figure 8).

\section{Discussion}

There is increasing evidence that Wnt signaling is subject to feedback regulation at various levels. For example, the $\beta$-catenin/TCF complex activates the promoter for full-length LEF1, indicating a positive feedback loop for Wnt signaling by inducing selective expression of full-length and $\beta$-catenin-sensitive forms of LEF/TCFs. ${ }^{9,21}$ Conductin/Axin2 creates a negative loop to silence the signaling pathway following transduction of Wnt signals. ${ }^{22}$ Moreover, deregulated $\beta$-catenin causes activation of $\mathrm{p} 53$, which feedbacks and downregulates $\beta$-catenin. ${ }^{23,24}$ We propose here that TCF4 should be added to the list of positive feedback activators of $\beta$-catenin-signaling.

Several lines of evidence from this study strongly support the agreement that upregulation of TCF4 expression occurs directly through transcriptional activation from the promoter mediated by $\beta$-catenin. First, endogenous TCF4 expression at both mRNA and protein levels was induced by stable overexpression of mutant $\beta$-catenin, as well as by LiCl treatment. Second, a 1.3-kb human TCF4 promoter fragment including a single consensus TCF/LEF-binding site fused to a luciferase reporter was activated by cotransfection of $\beta$-catenin and p300. Moreover, the induction could be largely inhibited by a dominant-negative form of TCF4, suggesting that the induction is mediated at the transcriptional level. Third, introduction of triple mutations into the TCF/LEF site in the promoter resulted in a decrease of the transactivation mediated by $\beta$-catenin $/ \mathrm{p} 300$, confirming that the site is critical for $\beta$-catenin-responsiveness. Finally, TCF4 expression apparently overlappped with nuclear $\beta$-catenin accumulation in Em Ca tissues, particularly in morular areas. We therefore conclude from the present data that a positive feedback loop of TCF4 expression mediated by $\beta$-catenin/p300 may be critical for trans-differentiation toward a squamoid phenotype in Em Ca cells, since transcriptional activation of target genes, such as p14 ${ }^{\mathrm{ARF}}$ and cyclin D1, is essential for this process (Figure 9). ${ }^{13}$ In contrast, the finding of ubiquitous TCF4 expression 

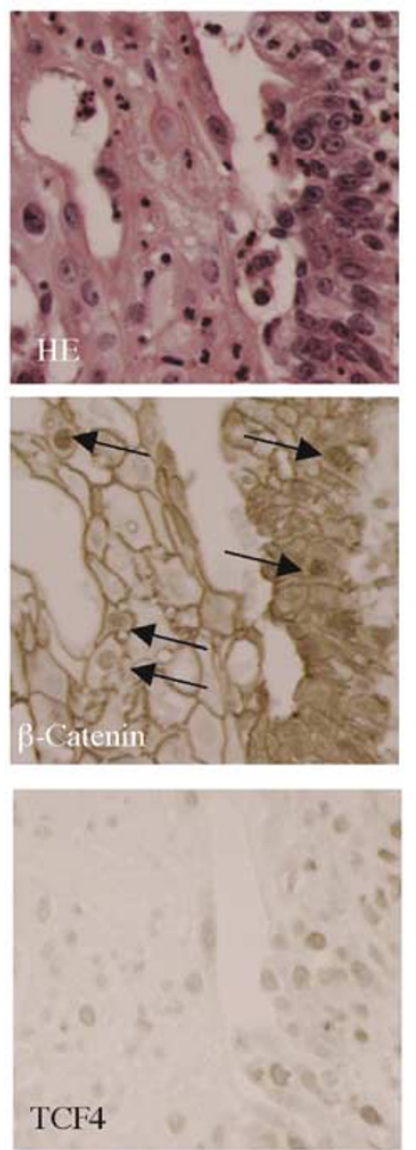

b

(\%) Nuclear $\beta$-catenin

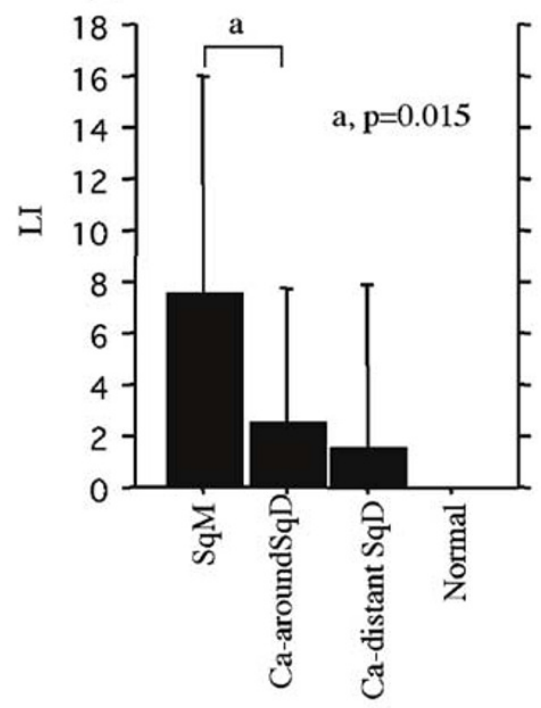

TCF4

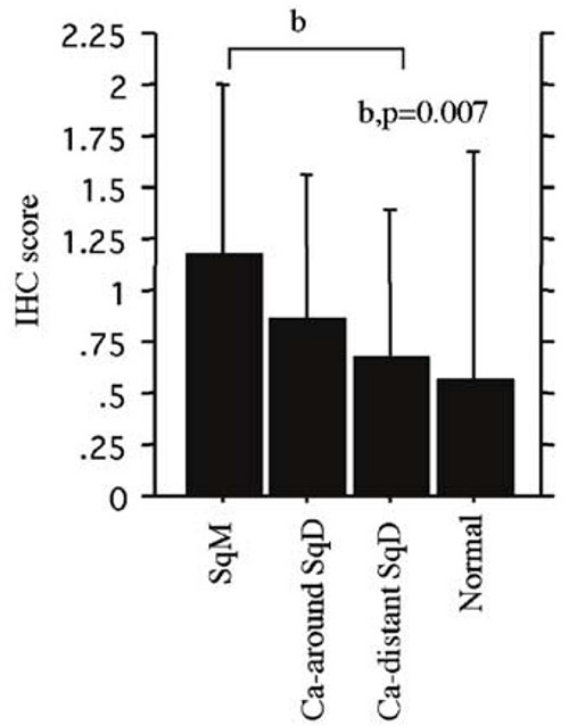

c

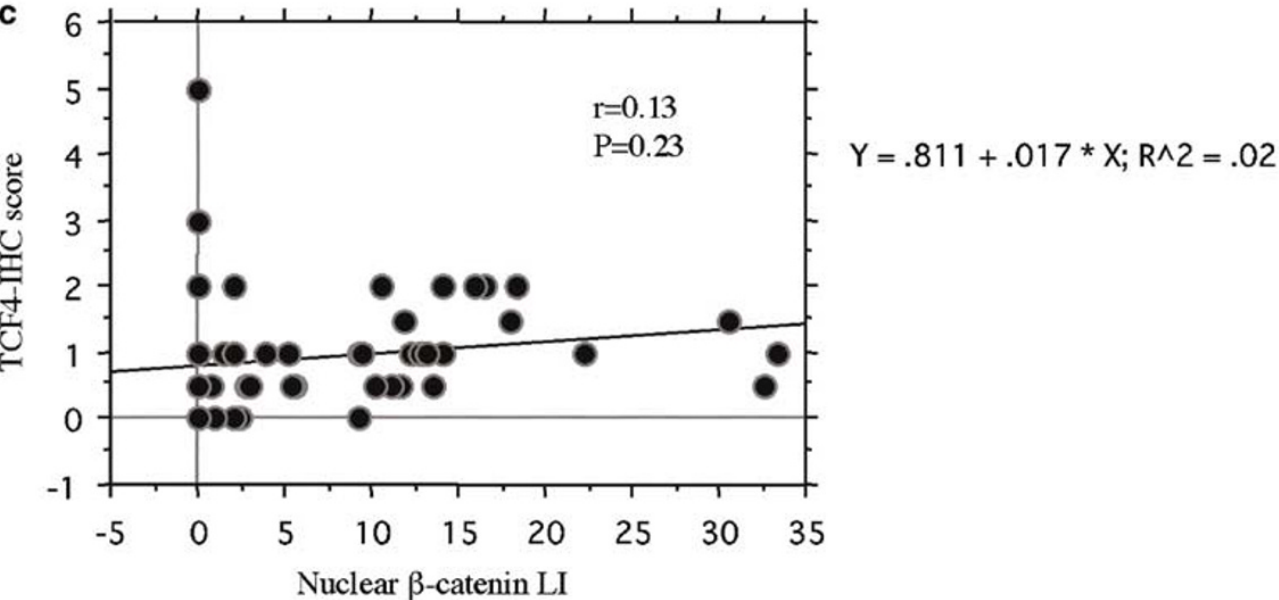

Figure 7 (a) Serial sections of an Em Ca with an area of squamous metaplasia (SqM). Staining is for H\&E, and immunohistochemically for $\beta$-catenin, and TCF4. Note the sporadic and weak nuclear staining for $\beta$-catenin (indicated by arrows) and TCF4 in SqM areas, as well as the surrounding glandular components. Original magnification, $\times 400$. (b) LIs and IHC scores for nuclear $\beta$-catenin and TCF4 in Em Cas with SqM areas. Data are means \pm s.d. values. (c) Correlation between nuclear $\beta$-catenin LIs and TCF4-IHC scores for Em Cas with SqM areas. $r$, Pearson's correlation coefficient.

in stromal cells lacking nuclear $\beta$-catenin allows us to speculate other regulatory mechanisms for this factor. Further studies of this point are clearly warranted.

Although activation levels of TCF4 triggered by $\beta$-catenin alone were relatively weak, the p300 coactivator cooperated strongly with $\beta$-catenin to specifically activate the promoter, suggesting that $\beta$-catenin recruits p300 as a coactivator for efficient transactivation during trans-differentiation. This conclusion is supported by the evidence that: (1) exogenous overexpressing $\beta$-catenin interacts with endogenous p300 in Ishikawa cells; (2) complexes showed active transcription as determined by onehybrid assay; and (3) endogenous p300 detected by immunohistochemistry was expressed ubiquitously in Em Cas, as well as normal endometrial tissues (data not shown).
Previous studies have generated conflicting findings for putative domains of interaction between p300/CBP (CREB-binding protein) and $\beta$-catenin, including (a) the CH-3 domain of p300 and Cterminal residues $630-781$ of $\beta$-catenin, ${ }^{14}$ (b) $\mathrm{CH} 1$ of p300 and N-terminal residues 48-217 of $\beta$-catenin, ${ }^{25}$ (c) the CREB-binding site (451-682) of CBP and the arm repeat 10 to the $\mathrm{C}$-terminus of $\beta$-catenin, ${ }^{26}$ and (d) $\mathrm{CH} 3$ of CBP and both $\mathrm{N}$ - and C-terminal arm repeats of $\beta$-catenin. ${ }^{27}$ One possible reason for the observed discrepancies may be the different strategies applied based on in vitro-synthesized p300 and $\beta$-catenin proteins with various truncating mutations. Conversely, it is possible that there are multiple and dispersed contacts between the two molecules. Our present findings demonstrate that $\beta$-catenin can interact predominantly with the 

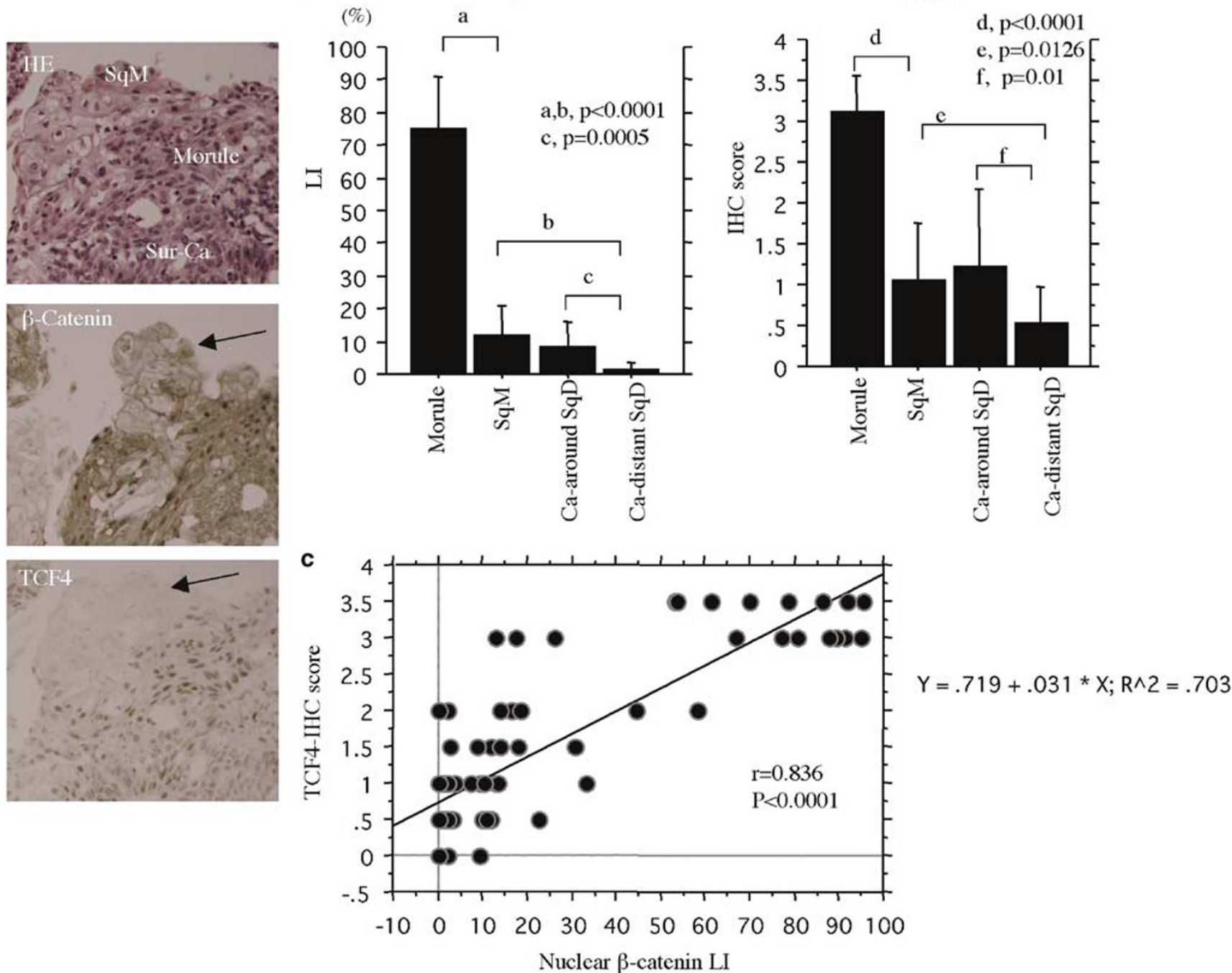

Figure 8 (a) Sequential changes in morphology from morular to squamous metaplastic (SqM) phenotypes in a trans-differentiated area of a Em Ca. Note the decrease in immunoreactivity for nuclear $\beta$-catenin and TCF4 in the SqM (indicated by the arrows) as compared to morular cells. Original magnification, $\times 200$ (b) LIs and IHC scores for nuclear $\beta$-catenin and TCF4 in morular and SqM areas, Ca-around $\mathrm{SqD}$, and Ca-distant SqD of tumors with coexistence of two squamoid phenotypes. Data are means \pm s.d. values. (c) Correlation between nuclear $\beta$-catenin LIs and TCF4-IHC scores for Em Cas with two phenotypes. r, Pearson's correlation coefficient

N-terminus of p300 in Em Ca cells in vivo, leading to formation of several enlarged dots in nuclei.

The histone acetyltransferase (HAT) activity of p300 is known to play an important role in gene transcription. ${ }^{28}$ For example, acetylation of specific lysine residues in the $\mathrm{C}$-terminus of p53 increases the sequence-specific DNA binding by altering the conformation of an inhibitory regulatory domain. ${ }^{29}$ In the present study, however, the $\mathrm{N}$-terminus of p300 proved sufficient to enhance $\beta$-catenin-dependent transcription in Ishikawa cells, despite the absence of the HAT domain, indicating the possibility that it can influence $\beta$-catenin-dependent transactivation without acetylation of histone or nonhistone proteins, in line with the report that p300 contains transcriptional activation domains that function independently of HAT. ${ }^{28}$
Another interesting finding in this study is that in Em Cas, expression of both nuclear $\beta$-catenin and TCF4 disappears during the change in morphology from morular to SqM cells. The morule is considered to represent an immature differentiation phenotype as compared to SqM areas, on the basis of investigation of bcl-2 expression. ${ }^{30}$ In addition, downregulation of the $\beta$-catenin-TCF pathway results in the promotion of a more-differentiated phenotype of colonic epithelial cells. ${ }^{31}$ It is likely, therefore, that the $\beta$-catenin-TCF4 signaling pathway plays an additional role in the maturation program leading toward the terminal squamoid phenotype of Em Cas (Figure 9).

In our previous studies, Em Cas in patients receiving prolonged progesterone therapy demonstrated an increase in the percentage of SqD areas, 


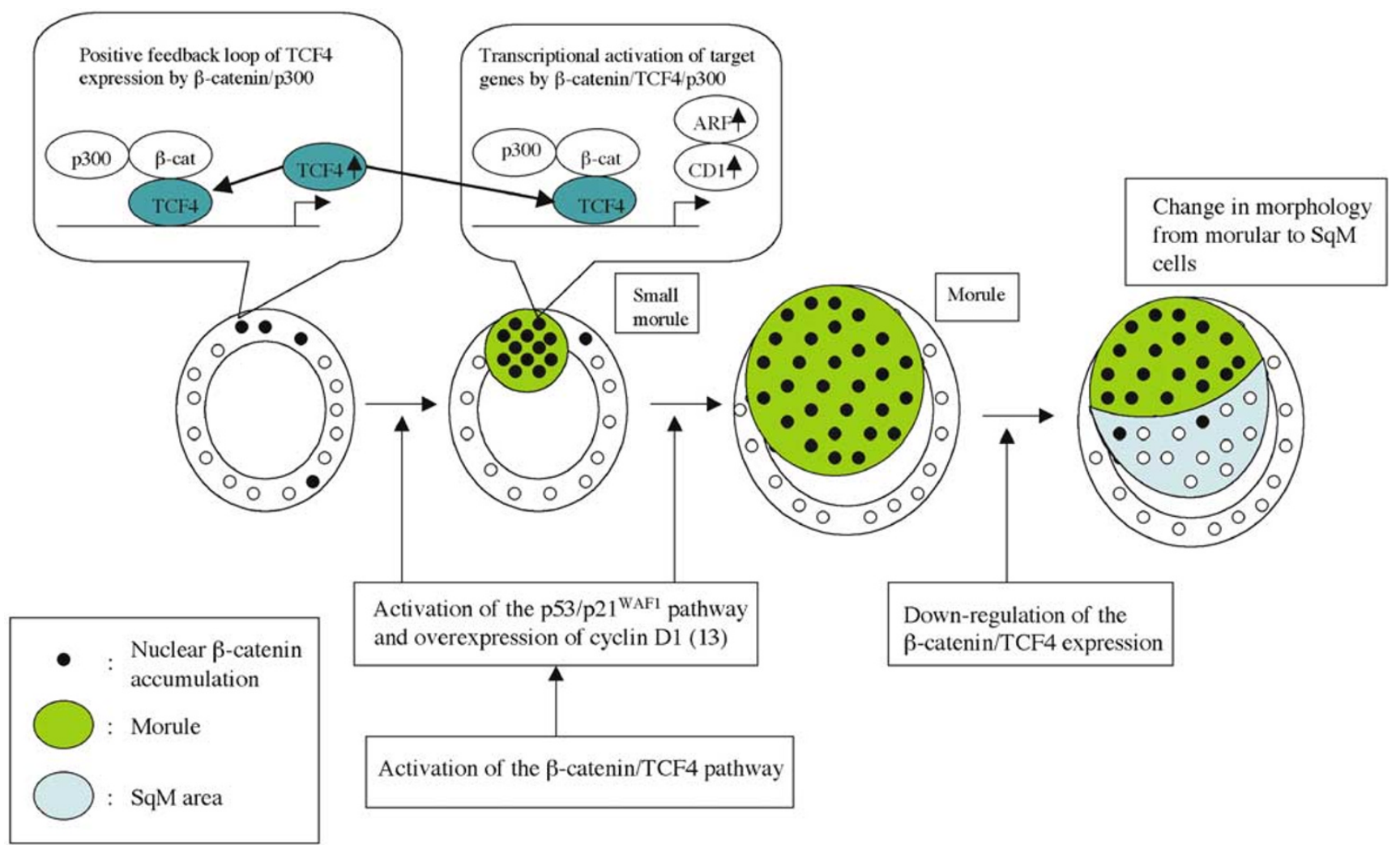

Figure 9 A model for the roles of $\beta$-catenin/TCF4/p300 during trans-differentiation toward a squamoid phenotype in Em Ca cells. Excess nuclear $\beta$-catenin interacts with TCF4, leading to upregulation of TCF4 expression through transcriptional activation from the promoter (positive feedback loop), this being enhanced by the p300 coactivator. Increased $\beta$-catenin/TCF4/p300 complexes induced transactivation of the target genes, such as $\mathrm{p} 14^{\mathrm{ARF}}$ and cyclin D1, resulting in induction of the trans-differentiation toward morular phenotypes of Em Ca cells. In enlarged morules, downregulation appears to be a signal for a more-differentiated squamoid phenotype (SqM).

leading to suppression of cell proliferation. ${ }^{32}$ Interestingly, stabilization of $\beta$-catenin in nuclei was evident during the therapy, being closely linked with the alteration in tumor morphology due to progesterone. ${ }^{33}$ Given the reported association between such abnormalities and several favorable prognostic factors for Em Cas, ${ }^{16}$ our observations in this study may provide a clue to understanding the biological significance of the $\beta$-catenin/TCF4 pathway in the switch in morphology from glandular to squamoid features in Em Cas.

In conclusion, the present study clearly demonstrated a positive feedback loop of TCF4 expression mediated by $\beta$-catenin/p300, implying a functional role during trans-differentiation toward a squamoid phenotype of Em Ca cells.

\section{Acknowledgements}

We are grateful to Drs Y Nakamura, RH Goodman, Y Nakatani, OK Engeland, and S Hirohashi for generous gifts of the expression and reporter plasmids used in this study. This study was supported by a grant from The Ministry of Educa- tion, Culture, Sports, Science and Technology of Japan (\#15590313).

\section{References}

1 Cadigan KM, Nusse R. Wnt signaling: a common theme in animal development. Genes Dev 1997;11: 3286-3305.

2 Korinek V, Barker N, Morin PJ, et al. Constitutive transcriptional activation by a $\beta$-catenin-Tcf complex in $\mathrm{APC}^{-1-}$ colon carcinoma. Science 1997;275: 1784-1787.

3 Morin PJ, Sparks AB, Korinek V, et al. Activation of $\beta$-catenin-Tcf signaling in colon cancer by mutations in $\beta$-catenin or APC. Science 1997;275:1787-1790.

4 Rubinfeld B, Robbins P, El-Gamil M, et al. Stabilization of $\beta$-catenin by genetic defects in melanoma cell lines. Science 1997;275:1790-1792.

5 Clevers $\mathrm{H}$, van de Wetering M. TCF/LEF factor earn their wings. Trends Genet 1997;13:485-489.

6 Eastman Q, Grosschedl R. Regulation of LEF-1/TCF transcriptional factors by Wnt and other signals. Curr Opin Biol 1999;11:133-240.

7 Roose J, Molenaar M, Peterson J, et al. The Xenopus Wnt effector XTcf-3 interacts with Groucho-related transcriptional repressor. Nature 1999;395:608-612. 
8 Hecht A, Stemmler MP. Identification of a promoterspecific transcriptional activation domain at the $\mathrm{C}$ terminus of the Wnt effector protein T-cell factor 4 . J Biol Chem 2003;278:3776-3785.

9 Hovanes K, Li TWH, Munguia JE, et al. $\beta$-Cateninsensitive isoforms of lymphoid enhancer factor- 1 are selectively expressed in colon cancer. Nat Genet 2001; 28:53-57.

10 Zaino RJ, Kurman R. Squamous differentiation in carcinoma of the endometrium: a critical appraisal of adenoacanthoma and adenosquamous carcinoma. Semin Diagn Pathol 1988;5:154-171.

11 Zaino RJ, Kurman R, Herbold D, et al. The significance of squamous differentiation in endometrial carcinoma: data from a Gynecologic Oncology Group Study. Cancer 1991;68:2293-2302.

12 Saegusa M, Okayasu I. Frequent nuclear $\beta$-catenin accumulation and associated mutations in endometrioid-type endometrial and ovarian carcinomas with squamous differentiation. J Pathol 2001;194:59-67.

13 Saegusa M, Hashimura M, Kuwata T, et al. $\beta$-Catenin simultaneously induces activation of the p53p21WAF1 pathway and overexpression of cyclin D1 during squamous differentiation of endometrial carcinoma cells. Am J Pathol 2004;164:1739-1749.

14 Hecht A, Vleminckx K, Stemmler MP, et al. The p300/ CBP acetyltransferases function as transcriptional coactivators of $\beta$-catenin in vertebrates. EMBO J 2000; 19:1839-1850.

15 Okudela K, Hayashi $\mathrm{H}$, Ito T, et al. K-ras gene mutation enhances motility of immortalized airway cells and lung adenocarcinoma cells via Akt activation: possible contribution to non-invasive expansion of lung adenocarcinoma. Am J Pathol 2004;164:91-100.

16 Saegusa M, Hashimura M, Yoshida T, et al. $\beta$-Catenin mutations and aberrant nuclear expression during endometrial tumorigenesis. Br J Cancer 2001;84: 209-217.

17 Nishida M, Kasahara K, Kaneko M, et al. Establishment of a new human endometrial adenocarcinoma cell line, Ishikawa cells, containing estrogen and progesterone receptors. Acta Obstet Gynec Jpn 1985;37:1103-1110.

18 Schmelz EM, Roberts PC, Kustin EM, et al. Modulation of intracellular $\beta$-catenin localization and intestinal tumorigenesis in vivo and in vitro by sphingolipids. Cancer Res 2001;61:6723-6729.

19 Orford K, Crockett C, Jensen JP, et al. Serine phosphorylation-regulated ubiquitination and degradation of $\beta$-catenin. J Biol Chem 1997;272:24735-24738.
20 Tetsu O, McCormick F. $\beta$-Catenin regulates expression of cyclin D1 in colon carcinoma cells. Nature 1999; 398:422-426.

21 Filali M, Cheng N, Abbott D, et al. Wnt-3A/ $\beta$-catenin signaling induces transcription from the LEF-1 promoter. J Biol Chem 2002;277:33398-33410.

22 Lustig B, Jerchow B, Sachs M, et al. Negative feedback loop of Wnt signaling through upregulation of conductin/axin2 in colorectal and liver tumors. Mol Cell Biol 2002;22:1184-1193.

23 Damals A, Kahan S, Shtutman M, et al. Deregulated $\beta$-catenin induces a p53- and ARF-dependent growth arrest and cooperates with Ras in transformation. EMBO J 2001;17:4912-4922.

24 Sadot E, Geiger B, Oren M, et al. Down-regulation of $\beta$-catenin by activated p53. Mol Cell Biol 2001;21: 6768-6781.

25 Sun Y, Kolligs FT, Hottiger MO, et al. Regulation of $\beta$-catenin transformation by the p300 transcriptional coactivator. Proc Natl Acad Sci USA 2000;97: 12613-12618.

26 Takemura K, Moon RT. The transcriptional coactivator $\mathrm{CBP}$ interacts with $\beta$-catenin to activate gene expression. J Cell Biol 2000;149:249-254.

27 Miyagishi M, Fujii R, Hatta M, et al. Regulation of Lef-mediated transcription and p53-dependent pathway by associating $\beta$-catenin with $\mathrm{CBP} / \mathrm{p} 300$. J Biol Chem 2000;275:35170-35175.

28 Goodman RH, Smolik S. CBP/p300 in cell growth, transformation, and development. Genes Dev 2000;14: 1553-1577.

$29 \mathrm{Gu}$ W, Roeder RG. Activation of p53 sequence-specific DNA binding by acetylation of the p53 C-terminal domain. Cell 1997;90:595-606.

30 Saegusa M, Okayasu I. Down-regulation of bcl-2 expression is closely related to squamous differentiation and progesterone therapy in endometrial carcinomas. J Pathol 1997;182:429-436.

31 Mariadason JM, Bordonaro M, Aslam F, et al. Downregulation of $\beta$-catenin TCF signaling is linked to colonic epithelial cell differentiation. Cancer Res 2001; 61:3465-3471.

32 Saegusa M, Okayasu I. Progesterone therapy for endometrial carcinoma reduces cell proliferation but does not alter apoptosis. Cancer 1998;83:111-121.

33 Saegusa M, Hamano M, Kuwata T, et al. Up-regulation and nuclear localization of $\beta$-catenin in endometrial carcinoma in response to progesterone therapy. Cancer Sci 2003;94:103-111. 